On peut parfois forcer les choses, en politique, tenter des jeux de pouvoir ... et gagner; mais il est probablement beaucoup plus important encore de savoir repérer les synergies, les conjonctions possibles, et les partenaires de bonne volonté.

L'élaboration en commun avec la Conférence des directeurs cantonaux de la santé (CDS) d'un projet de révision de la LAMal qui permette la fin de la clause du besoin sans ouvrir la porte au chaos, est un exemple de collaboration fructueuse.

Les cantons sont constitutionnellement responsables de la fourniture de soins à la population, mais ils sont surtout proches du terrain, et - ce n'est pas à négliger! - leurs dirigeants ont, à chaque élection, des comptes à rendre à la population.
Leur donner un rôle plus précis dans la définition de notre système de santé, après la clause du besoin, est donc aux yeux de la FMH une voie de bon sens, d'autant que les médecins, connaisseurs de la médecine et du terrain sur lequel elle s'exerce, seront associés sinon aux décisions, tout au moins aux réflexions qui les précéderont.

Hans-Peter Kuhn, Secrétaire général adjoint de la FMH et responsable de notre service juridique, décrit ci-après plus en détail cet excellent projet de la FMH et de la CDS, qui est actuellement dans les mains de la Commission de la santé du Conseil des Etats.

Dr Jacques de Haller, Président de la FMH

\title{
Fin de la clause du besoin: une possibilité de régulation différenciée reste nécessaire
}

Instaurer une clause du besoin revenait à vouloir simplement geler la situation: par cette mesure, le législateur visait à maintenir le statu quo, ce qui n'est évidemment jamais une solution durable. Cela étant, mettre fin à la clause du besoin sans introduire une réglementation subséquente serait une option dangereuse. En effet, vu l'accord sur la libre circulation des personnes avec l'UE, de nombreux médecins seraient alors susceptibles d'ouvrir un cabinet, et peut-être pas dans des régions ou dans les disciplines où l'approvisionnement en soins le demande le plus. C'est pourquoi, de l'avis de la FMH et de la CDS, les cantons doivent pouvoir procéder à une régulation différenciée afin de garantir une fourniture de soins de haute qualité, accessible à tous et d'un coût abordable.

La proposition commune de la FMH et de la CDS prévoit la possibilité pour les cantons de réguler les ressources lorsqu'il existe un risque de sur- ou sous-approvisionnement en soins:

Idéalement, les médecins ouvrent un cabinet là où il existe une demande en soins. Dans un tel cas, nul besoin de régulation de la part des cantons.

En présence d'un risque de sur-approvisionnement, les cantons peuvent introduire une procédure d'admission (sinon, l'autorisation d'exercer suffit, comme avant 2002) et décider, en fonction de la demande, à quel endroit et dans quelle spécialité (médecine de premier recours, gynécologie, psychiatrie, etc.) il est possible d'ouvrir des cabinets médicaux.

S'il y a risque de sous-approvisionnement - un scénario réaliste dans quelques disciplines - les pouvoirs publics ne doivent pas non plus rester inactifs. La Confédération devrait aider à éviter ou maîtriser les carences en collaborant au financement de postes d'assistant en cabinet et, si nécessaire, en admettant l'installation de médecins venant de l'extérieur de l'EU. Et dans les régions insuffisamment desservies, les cantons peuvent aussi, en cas de nécessité, soutenir l'établissement de médecins par des incitations économiques au niveau des frais d'investissement ou d'exploitation. Il est intéressant de constater que l'ancienne LAMA connaissait déjà une possibilité de verser des indemnités à des médecins pour leur permettre de s'installer dans des régions de montagne retirées, où leur présence était nécessaire pour assurer les soins mais où ils ne parvenaient pas à couvrir les coûts d'un cabinet en pratiquant le tarif normal des caisses. Lors des débats sur la LAMal introduite par la suite, personne n'envisageait plus qu'une pénurie de médecins puisse intervenir à nouveau dans certaines disciplines ou régions - une erreur d'appréciation, comme nous le savons aujourd'hui.

Dans le projet présenté, le canton consulte la société cantonale de médecine et l'association des assistants et chefs de clinique. La décision reste cependant exclusivement de la compétence du canton. Des modèles de ce genre ont déjà fait leurs preuves: pour rendre ses décisions, le canton de Genève s'appuie par exemple sur une commission consultative réunissant des représentants de la société de médecine, des médecins-assistants et des hôpitaux.

Hanspeter Kuhn, avocat Responsable du service juridique de la FMH Secrétaire général adjoint de la FMH 